\title{
Tiña Capitis en niños
}

\section{Tinea capitis in children}

\author{
Natalia Vargas-Naviaa , Geovanna A. Ayala Monroy ${ }^{\mathrm{b}}$, Catalina Franco Rúa ${ }^{\mathrm{b}}$, \\ Juan Pablo Malagón Caicedoc, Juan Pablo Rojas Hernández ${ }^{\mathrm{d}}$
}

\author{
aDermatóloga Pediatra. Universidad del Valle. Fundación Clínica Infantil Club Noel. Cali, Colombia \\ bResidente, Sección de Dermatología, Facultad de Salud. Universidad del Valle. Cali, Colombia \\ 'Médico, Universidad Libre Seccional Cali, Colombia \\ dPediatra Infectólogo, Universidad del Valle. Cali, Colombia. Grupo de Investigación en Pediatría-GRINPED COL 0142019. \\ Fundación Clínica Infantil Club Noel. Universidad Libre Seccional Cali, Colombia
}

Recibido: 25 de julio de 2019; Aceptado: 13 de abril de 2020

\section{¿Qué se sabe del tema que trata este estudio?}

La tiña capitis (TC) o tiña del cuero cabelludo es una infección micótica causada por dermatofitos principalmente de los géneros Microsporum y Trichophyton. Constituye una causa de consulta frecuente en pediatría y suele manifestarse con áreas alopécicas, descamación y cambios inflamatorios variables.

\begin{abstract}
¿Qué aporta este estudio a lo ya conocido?
Los agentes causales presentan una distribución geográfica dinámica. La dermatoscopia y la luz de Wood son útiles para la aproximación diagnóstica. El agente causal, la forma de invasión micótica y el estado inmunológico del huésped determinan la presentación clínica. La tricotilomanía y alopecia areata son diagnósticos diferenciales. Los antimicóticos sistémicos combinados con medidas locales están indicados.
\end{abstract}

\section{Resumen}

La tiña capitis (TC) es una infección por dermatofitos con una alta prevalencia en la población pediátrica. Su epidemiología ha cambiado en las últimas décadas debido a la creciente migración poblacional alrededor del mundo. Se han identificado factores de riesgo ambientales y propios del huésped, relacionados al desarrollo de esta infección. Las manifestaciones clínicas son variables y dependen del agente causal. La dermatoscopia y la luz de Wood son herramientas útiles para la aproximación diagnóstica, sin embargo, la confirmación de la infección se basa en las pruebas micológicas. El aislamiento del agente causal permite orientar el tratamiento antifúngico adecuado, siendo estos eficaces y seguros en población pediátrica. El tratamiento se centra en la terapia antimicótica sistémica en combinación con medidas locales. El objetivo del manuscrito es hacer una revisión actualizada sobre el enfoque clínico y terapéutico de la TC en población pediátrica.

\section{Palabras clave:}

Tiña capitis; dermatofitosis; epidemiología; clínica; tratamiento 


\begin{abstract}
Tinea capitis (TC) is a dermatophyte infection with a high prevalence in the pediatric population. Its epidemiology has changed in recent decades due to increasing population migration worldwide. Environmental and host-specific risk factors have been identified which are with the development of this infection. The clinical manifestations are variable and depend on the causal agent. Dermatoscopy and Wood's lamp are useful tools for the diagnostic approach; however, the confirmation of infection is based on mycological tests. The identification of the causal agent allows guiding the appropriate antifungal treatment, which is specific and safe in the pediatric population. Treatment focuses on systemic antifungal therapy combined with local measures. The objective of this paper is to carry out an updated review of the clinical and therapeutic approach to TC in the pediatric population.
\end{abstract}

\section{Keywords:}

Tinea capitis;

Dermatophytosis;

Clinical features;

Epidemiology;

Treatment

\section{Introducción}

Las dermatofitosis o tiñas son infecciones frecuentes causadas por un grupo de hongos filamentosos que tienen la capacidad de invadir los tejidos queratinizados (piel uñas y pelo), que pertenecen a los géneros Epidermophyton spp, Microsporum spp y Trichophyton $s p p^{1}$.

La Tiña capitis (TC), también conocida como tiña de la cabeza o cuero cabelludo, constituye una de las infecciones fúngicas más comunes en la población infantil ${ }^{1,2}$. Se define como una micosis superficial de la piel, el cuero cabelludo o los folículos pilosos de la cabeza, causada por dermatofitos principalmente de los géneros Microsporum spp y Trichophyton $s p p^{1,3}$. Las manifestaciones clínicas son variables, dependen del agente etiológico y la respuesta inmunológica de cada huésped. El diagnóstico se basa en los hallazgos clínicos, la fluorescencia con luz de Wood y el estudio dermatoscópico, sin embargo, la confirmación de la infección se realiza mediante el examen directo con hidróxido de potasio e identificación del microorganismo a través del cultivo micológico ${ }^{4}$.

El objetivo del manuscrito es hacer una revisión actualizada sobre el enfoque clínico y terapéutico de la TC en población pediátrica.

\section{Epidemiología}

La TC es una infección que afecta principalmente a la población pediátrica $(97 \%)^{3,5}$, con una mayor prevalencia entre los 3 y 7 años $^{6}$, predominio en zonas rurales y suburbanas. En estas poblaciones, los factores relacionados con una mayor frecuencia de esta infección son el nivel socioeconómico bajo, familias numerosas, el hacinamiento y las pobres condiciones de higiene $e^{3,5,7,8}$.

La epidemiología de la TC varía dependiendo de la localización geográfica y la población estudiada. En Estados Unidos de América (EE.UU.), se reportó una prevalencia del $0 \%$ al $19,4 \%$ en población escolar 9 Otros autores han descrito prevalencia en escolares de 0,23\% en Barcelona, 2,5\% en Londres, y $13-31,2 \%$ en Nigeria $^{10}$.

Los agentes causales tienen una distribución geográfica dinámica, producida en gran medida por la migración de poblaciones portadoras de un lugar a otro del planeta. Se ha descrito que las infecciones por Trichophyton tonsurans son más comunes en América Central, EE.UU. y en partes de Europa Occidental, mientras que por Microsporum canis se observan principalmente en Suramérica ${ }^{3,8,11}$, Centro y Sur de Europa, África Occidental y Oriente Medio ${ }^{12,13}$. Este patrón de distribución se ha visto modificado por los movimientos migratorios en Europa, EE. UU. y Sudamérica. La incidencia de infecciones por Trichophyton tonsurans se ha incrementado en regiones como EE.UU., Europa Occidental, Reino Unido, Francia e India ${ }^{14-16}$. Por otra parte, ocasionalmente se han registrado brotes en áreas no endémicas de Trichophyton rubrum, Trichophyton mentagrophytes, Trichophyton violaceum y Trichophyton soudanense, asociado a migraciones desde Mediterráneo, Europa del Este, África y Asia Central ${ }^{17,18}$. Recientemente, en Montreal, Canadá, Marcoux y colaboradores $^{19}$, describieron un incremento en los últimos 17 años, de infecciones causadas por especies africanas (M. audouinii, T. soudanense, T. violaceum, y T. schoenleinii).

En Latinoamérica, diferentes estudios epidemiológicos sobre TC en México, Guatemala, Chile, Colombia y Brasil han identificado como principal agente causal a $M$. canis $^{3,8,20-22}$. Sin embargo, se ha descrito la emergencia de agentes como M. audouinii, T. tonsurans y Microsporum gypseum, en algunos países del Caribe y Suramérica ${ }^{23-26}$.

\section{Etiología}

Los dermatofitos causantes de la TC constituyen un grupo de hongos estrechamente relacionados, capaces 
de invadir e infectar los tejidos queratinizados, dado que la queratinasa les permite parasitar el pelo, la piel y las uñas, tanto del hombre como de algunos animales. Producen infecciones generalmente superficiales, afectando en un principio al estrato córneo de la piel y sus $\operatorname{anexos}^{27}$.

Desde el punto de vista ecológico, los dermatofitos se agrupan en 3 categorías: antropofílicos, que sólo ocasionan micosis en el hombre; zoofílicos, que infectan animales y a partir de estos contagian al hombre; y geofílicos, que se encuentran en el suelo y se nutren de la queratina allí existente, desde donde pueden infectar las estructuras queratinizadas tanto de animales como del hombre. En general, estos últimos suelen provocar una menor respuesta inflamatoria en el huésped, generando lesiones crónicas (en la tabla 1 se recogen las especies más frecuentes de cada grupo $)^{27}$.

Por otra parte, estos dermatofitos se clasifican en tres géneros: Epidermophyton spp, Microsporum spp y Trichophyton spp. Actualmente, hay reconocidas más de 40 especies, entre ellas, alrededor de una docena son patógenas para el hombre (tabla 2$)^{7}$.

La TC es causada habitualmente por especies del género Microsporum y Trichopyton, siendo M. canis el principal agente causal. Se encuentran en menor proporción $T$. violaceum, $T$. tonsurans, $T$. soudanense y M. audouinii. Trichophyton rubrum, a pesar de ser el dermatofito más comúnmente aislado en el mundo, es una causa excepcional de TC, mientras que E. floccosum no parasita el pelo por lo que no es un agente causal de esta enfermedad ${ }^{27}$.

Tabla 1. Clasificación ecológica de los dermatofitos

\begin{tabular}{lll}
\hline Antropofílicos & Zoofílicos & Geofílicos \\
\hline M. audouinii & M. canis & M. gypseum \\
M. ferrogineum & T. mentagrophytes var. mentagrophytes & M. fulvum \\
T. rubrum & var. erinacei var. quinckeanum & M. nanum \\
T. schoenleinii & T. verrucosum & M. coockei \\
T. mentagrophytes var. Interdigitale & T. equinum & M. praecox \\
T. tonsurans & T. verrucosum & \\
T. violaceum & T. simii & \\
T. concentricum & M. equinum & \\
T. megninii & M. gallinae & \\
T. soudanense & M. nanum & \\
T. yaoundei & M. persicolor & \\
T. gourvilii & & \\
E. floccosum & & \\
\hline
\end{tabular}

Tabla 2. Especies de dermatofitos

\begin{tabular}{lll}
\hline Epidermophyton & Microsporum & Trichophyton \\
\hline E. floccosum & M. amazonicum & T. ajelloi \\
M. stockdale & M. boullardii & T. concentricum \\
M. canis & T. equinum \\
M. cookei & T. flavescens & T. georgiae \\
M. equinum & T. gloriae \\
M. ferrugineum & T. gourvilii \\
M. fulvum & T. longifusum \\
M. gallinae & T. mariatii \\
M. gypseum & T. megninii \\
M. panum & T. mentagrophytes \\
M. praecolor & T. rubrum \\
M. racemosum & T. schoenleinii \\
M. ripareae & T. simii \\
M. vanbreuseghemi & T. soudanense \\
& T. terrestre \\
& T. tonsurans \\
& T. vanbreuseghemii \\
& T. verrucosum \\
\hline
\end{tabular}




\section{Fisiopatología}

La TC ocurre por la invasión del tallo piloso por dermatofitos. Puede proceder de animales que transmiten la infección a humanos, conocidas como infecciones zoofílicas, que generalmente se acompañan de un componente inflamatorio, o por propagación de humano a humano, conocida como dermatofitosis antropofílica, que genera lesiones escasamente inflamatorias y de tipo persistente ${ }^{3}$.

Cuando ocurre el paso de estructuras micóticas de un huésped a otro, acontece la primera fase de invasión epidérmica o del tallo del pelo, que consiste en la adhesión entre las estructuras fúngicas, denominadas artroconidias y los queratinocitos. La penetración del pelo implica la producción de una gran variedad de enzimas proteolíticas, que funcionan en ambientes ácidos, alcalinos o neutros ${ }^{3}$. Entre ellas se destaca el papel de las queratinasas, que le permiten al hongo penetrar el tejido queratinizado, posterior a lo cual, las hifas invaden el estrato córneo, la queratina y se extienden hacía la periferia.

Una vez el cuerpo reconoce al microorganismo invasor, desencadena una respuesta inmune innata para intentar controlar la infección. Como parte de esta respuesta se encuentran la expresión de péptidos antimicrobianos producidos naturalmente, incluidas las b-defensinas humanas (hBD), la catelicidina LL-37 y la dermicidina ${ }^{28}$. Estos péptidos juegan un papel importante en la protección contra las infecciones de la piel, incluidos los dermatofitos. También, se ha descrito que la infección por Microsporum canis desencadena una rápida secreción de IL-1b (vía activación del inflamosoma $)^{29}$.

Otro mecanismo innato de defensa, importante en el cuero cabelludo, incluye la inhibición del crecimiento de dermatofitos por parte de los ácidos grasos saturados con longitudes de cadena de 7, 9, 11 y 13 residuos de carbono. También están involucrados mecanismos de defensa relacionados principalmente con la activación de neutrófilos y macrófagos ${ }^{23,30}$. Adicionalmente la activación de las células $\mathrm{T}$ es un evento clave para limitar la infección, tanto el perfil Th1 como Th17. Todo esto con el fin de retrasar el desarrollo del microorganismo o de volver el ambiente no favorable para este. La respuesta inflamatoria varía entre los individuos y tiende a ser mucho más severa en infecciones por especies zoofílicas 7,31 .

Existen tres tipos principales de invasión del tallo del pelo, los cuales determinan parcialmente la presentación clínica:

- Endotrix: parasitación del hongo dentro del tallo piloso, las hifas crecen hacia abajo del pelo y penetran en la vaina de éste, dejando la cutícula intacta. De esta manera, las hifas se convierten en artro- conidios y pueden ser visualizados a la microscopía directa, este tipo de infección no fluórese a la luz de Wood. Clínicamente los pelos se rompen a nivel del cuero cabelludo, lo que deja "muñones" hinchados dentro de los folículos (tiña de puntos negro). Los agentes etiológicos que presentan este tipo de parasitación son principalmente: $T$. tonsurans, $T$. soudanense y $T$. violaceum, no obstante, otros agentes con este tipo de parasitación son T. gourvilli, T. yaoundei, y ocasionalmente T. rubrum $^{7,32}$.

- Ecto-endotrix: parasitación interna y externa, en la que el tallo del pelo se invade a nivel del folículo medio y las hifas crecen hacia el bulbo, de forma externa al folículo y cubren la superficie del pelo, en algunos casos se pueden desarrollar artroconidias por dentro y por fuera del pelo, en estos casos, las esporas que rodean pueden semejar la estructura de una vaina. La invasión del pelo tipo ectoendotrix se desarrolla de forma similar al tipo endotrix, con la excepción de que las hifas destruyen la cutícula, crecen alrededor de la vaina externa del pelo y las hifas se convierten en artroconidias infectantes. Se asocia a una presentación clínica en placas alopécicas, con escamas y pelos que se rompen 2 a $3 \mathrm{~mm}$ o más por encima del nivel del cuero cabelludo. Es causada principalmente por Microsporum spp, como M. audouinii, M. canis, M. distortum, M. ferrugineum, M. gypseum, M. nanum y también $T$. verrucosum (figura 1) ) $^{7,32}$.

- Favus: Los espacios de aire en los tallos del cabello son característicos y las hifas fúngicas forman grandes grupos en la base de los pelos, donde entran en el folículo al nivel de la epidermis. Es un tipo de tiña inflamatoria, se caracteriza por costras queratósicas amarillas elevadas, en forma de «cazoleta», alrededor de los folículos pilosos, que contienen hifas y pueden ser altamente infecciosa; los pelos afectados están menos dañados que en los otros tipos y pueden continuar creciendo hasta alcanzar una longitud considerable. Su principal agente causal es T. schoenleinii un dermatofito antropofílico. En la tabla 3, se muestra la correlación entre el agente causal y las manifestaciones clínicas de la $\mathrm{TC}^{32}$.

\section{Manifestaciones clínicas}

Existen dos formas de presentación clínica: seca e inflamatoria. La TC seca se manifiesta con descamación, pelos cortos (2-3 mm), gruesos, deformados y ocasionalmente cubiertos por una vaina blanquecina. Esta forma seca, a su vez, se subdivide en dos variantes: microspórica y tricofítica. La variante microspóri- 


\section{Parasitación ecto-endotrix}

Parasitación endotrix

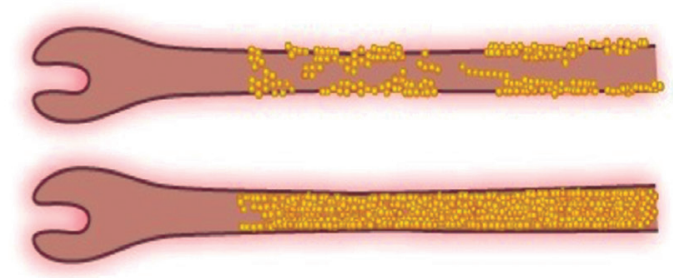

Figura 1. Distribución de esporas: ecto-endottrix y endotrix.

Tabla 3. Organismos asociados con formas clínicas de la tiña capitis

\begin{tabular}{llll}
\hline Inflamatoria & No inflamatoria & Puntos negros & Favus \\
\hline M. audouinii & M. audouinii & T. tonsurans & M. gypseum \\
M. canis & M. canis & T. violaceum & T. schoenleinii \\
M. gypseum & M. ferrugineum & & \\
T. violaceum & T. onsurans & & \\
M. nanum & & & \\
T. mentagrophytes & & & \\
T. schoenleinii & & & \\
T. tonsurans & & & \\
T. verrucosum & & & \\
\hline
\end{tabular}

ca está relacionada con el género Microsporum spp y se manifiesta con una o varias placas grandes, con pelos cortos y rotos a un mismo nivel, que pueden confluir formando una sola placa de gran extensión, en caso de avance de la infección, puede progresar a descamación severa, ruptura de la cutícula y eventualmente traducirse en un pelo frágil y quebradizo ${ }^{31}$. El principal agente etiológico relacionado con esta forma clínica es $M$. canis, seguido de M. gypseum y M. audouinii. La variante tricofítica de la forma seca se caracteriza por alopecia difusa, con placas pequeñas e irregulares intercaladas con pelos sanos, el pelo afectado se rom- pe en la superficie y se puede observar la abertura del folículo, mostrando una apariencia de puntos negros ("granos de pólvora") (figura $2 \mathrm{~A}$ ). El principal agente causal de esta variante es: T.tonsurans, seguido de $T$. mentagrophytes y T. Rubrum ${ }^{7}$.

La forma inflamatoria de la tiña capitis o querion de Celso (figura 2 B y C) es principalmente causada por $M$. canis y T. tonsurans, se manifiesta con una placa granulomatosa, constituida por pústulas y abscesos múltiples, puede estar asociado a adenopatías regionales, fiebre, malestar general, dolor local y con frecuencia resuelve dejando un área de alopecia cicatrizal ${ }^{33}$.
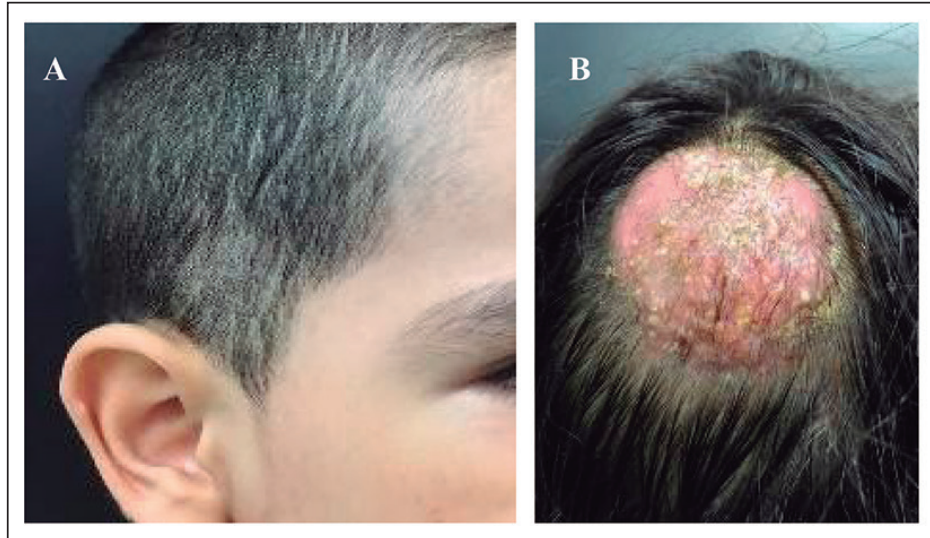

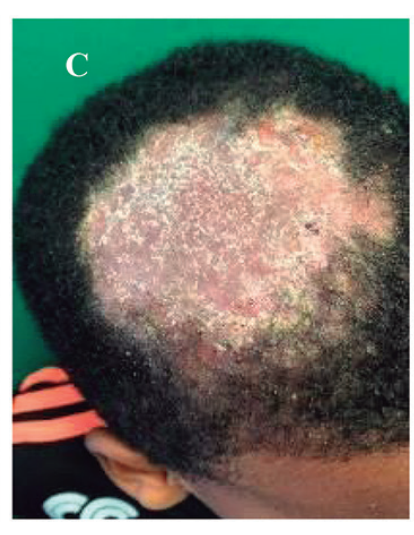

Figura 2. Presentación clínica de la tiña capitis $\mathbf{A}$. "Tiña de puntos negros". B-C. Querion de Celso. 


\section{Diagnóstico}

El diagnóstico de TC es clínico, la presencia de alopecia, escamas, inflamación folicular, adenopatía auricular y cervical posterior, son datos que sugieren una infección por dermatofitos, sin embargo, se recomienda realizar una confirmación micológica para orientar la terapia sistémica ${ }^{4,8}$. Dentro de la exploración física se cuenta con herramientas que facilitan el diagnóstico, tales como, la luz de Wood, siendo esta un apoyo técnico importante que proporciona orientación para la identificación del microorganismo involucrado. No obstante, en el caso de las tiñas tricofíticas no se presenta fluorescencia, por el predominio de la infección de tipo endotrix, a diferencia de la parasitación ectoendotrix apreciada en M. audouinii y $M$. canis los cuales produce la fluorescencia amarillo-verdoso característica (figura $3 \mathrm{~A}$ y $\mathrm{B}$ ) ${ }^{6,7}$.
Otra herramienta útil en el diagnóstico de esta patología infecciosa es la tricoscopía, una técnica no invasiva de evaluación del pelo y del cuero cabelludo, que puede realizarse a través de una pieza de mano (dermatoscopio) que magnifica $10 \mathrm{x}$, o mediante un videodermatoscopio que magnifica de 20x a 70x, permitiendo la visualización de patrones dermatoscópicos característicos de la TC tales como los pelos "en coma", "en sacacorchos", "en zigzag", "en clave Morse", los pelos cortos, los puntos negros, el eritema y la descamación perifolicular (figura 4 A y B), hallazgos que facilitan su diferenciación con otras patologías del cuero cabellu$\mathrm{do}^{34-39}$. Así mismo pueden orientar al clínico sobre la posible etiología infecciosa, teniendo en cuenta que los pelos en sacacorchos se han descrito mayormente en relación con infecciones por Trichophyton, y los pelos en clave Morse, los pelos en zigzag, los pelos retorcidos y la descamación difusa se han relacionado con ma-
Figura 3. Paciente con tiña capitis microspórica A. Placa descamativa circunscrita en cuero cabelludo B. Fluorescencia positiva a la evaluación con la luz de Wood.

Figura 4. A. Hallazgos tricoscópicos frecuentes en TC: puntos negros (flecha azul), pelos en sacacorchos (flecha roja), pelos rotos (flecha negra), pelos en "zigzag" (flecha naranja), pelos en coma (flecha verde) y eritema perifolicular (círculo negro). B. Otros cambios dermatoscópicos descritos en TC: descamación perifolicular (flecha blanca) y pelos en clave Morse (flecha amarilla).
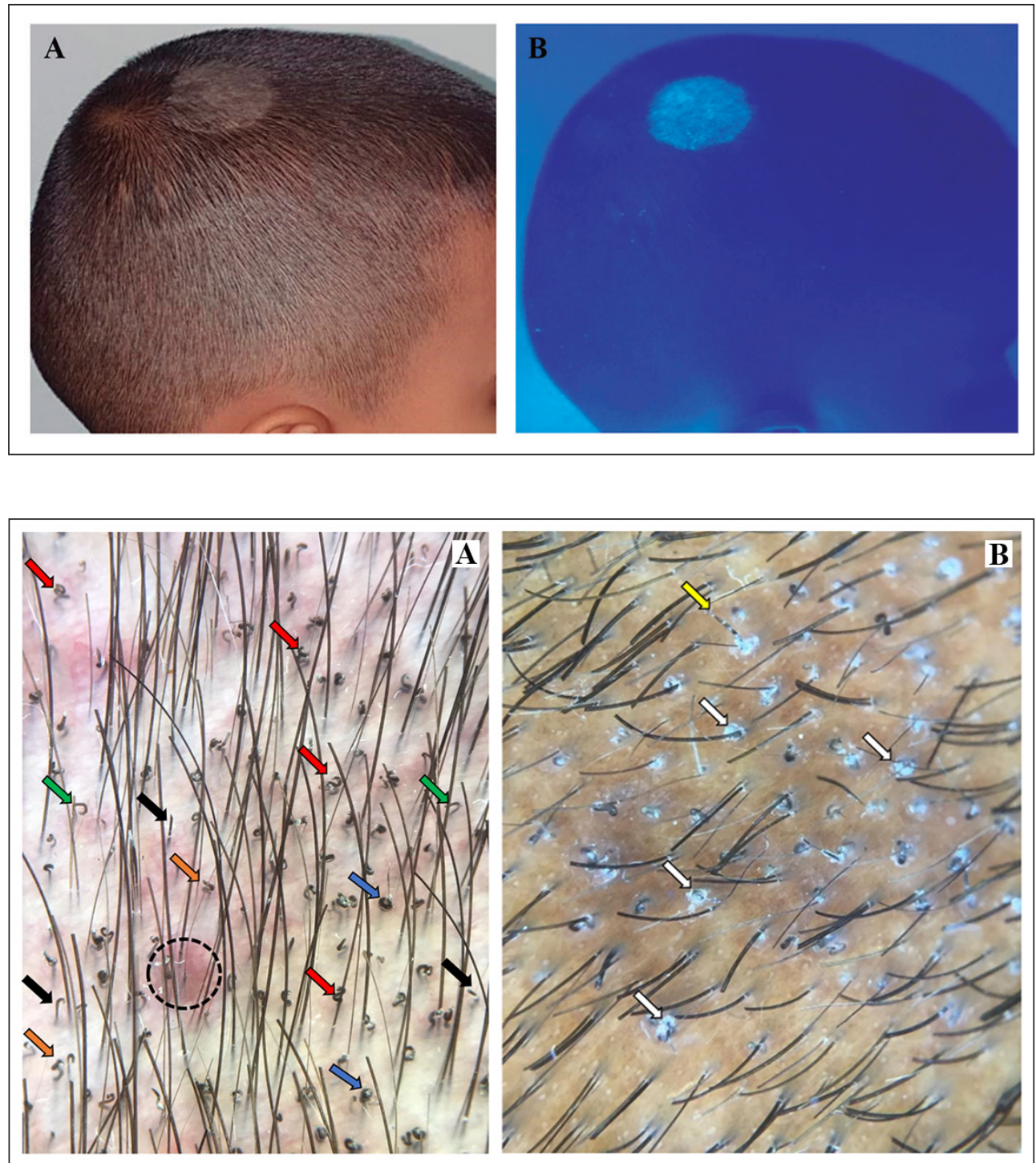
yor frecuencia a infecciones por Microsporum spp sp $^{39}$ Adicionalmente se ha descrito el papel de la tricoscopia en el seguimiento durante el tratamiento de la TC, considerándose un marcador tricoscópico de eficacia terapéutica, la desaparición de pelos distróficos (pelos en coma, pelos en sacacorchos, pelos en zigzag, pelos en clave Morse, pelos rotos y puntos negros), después de 4 a 12 semanas del inicio del tratamiento con una desaparición más tardía de otros signos como descamación perifolicular. Sin embargo estos hallazgos son orientativos y no son considerados marcadores de falla terapéutica ${ }^{39}$.

En todos los casos, la confirmación diagnóstica se debe realizar con estudio micológico (examen directo y cultivo), tomando la muestra de pelos rotos o puntos negros ${ }^{4}$. La muestra se obtiene retirando los pelos y la escama del cuero cabelludo con bisturí, por cepillado del cuero cabelludo, o con cytobrush (muestra por cepillo), que permite mejorar sensibilidad y tiempo para obtener un cultivo positivo, pero no permite examinar la muestra microscópicamen$\mathrm{te}^{7}$.

El examen directo se realiza con $\mathrm{KOH}+$ dimetilsulfóxido (DMSO) o negro de clorazol. En caso de contar con microscopio de fluorescencia, se puede hacer uso del blanco de calcoflúor. Se examina en búsqueda de hifas, artroconidias y esporas que pueden estar localizadas al interior del pelo (endotrix), siendo más común en infecciones por T. tonsurans; en caso de que la parasitación del pelo se encuentre alrededor del mismo (ecto-endotrix) se debe pensar en Microsporum spp (figura $5 \mathrm{~A}$ y B $)^{7}$.

El cultivo micológico se realiza en un medio de cultivo para hongos agar Sabouraud o que contenga antibióticos (Mycosel), con el fin de inhibir el crecimiento de mohos no dermatofitos. Los cultivos deben incubarse durante al menos dos semanas y el crecimiento de las colonias es evidente en una a seis semanas. Este es el método de elección en países en vía de desarrollo (figura $6 \mathrm{~A} \mathrm{y} \mathrm{B})^{7}$.
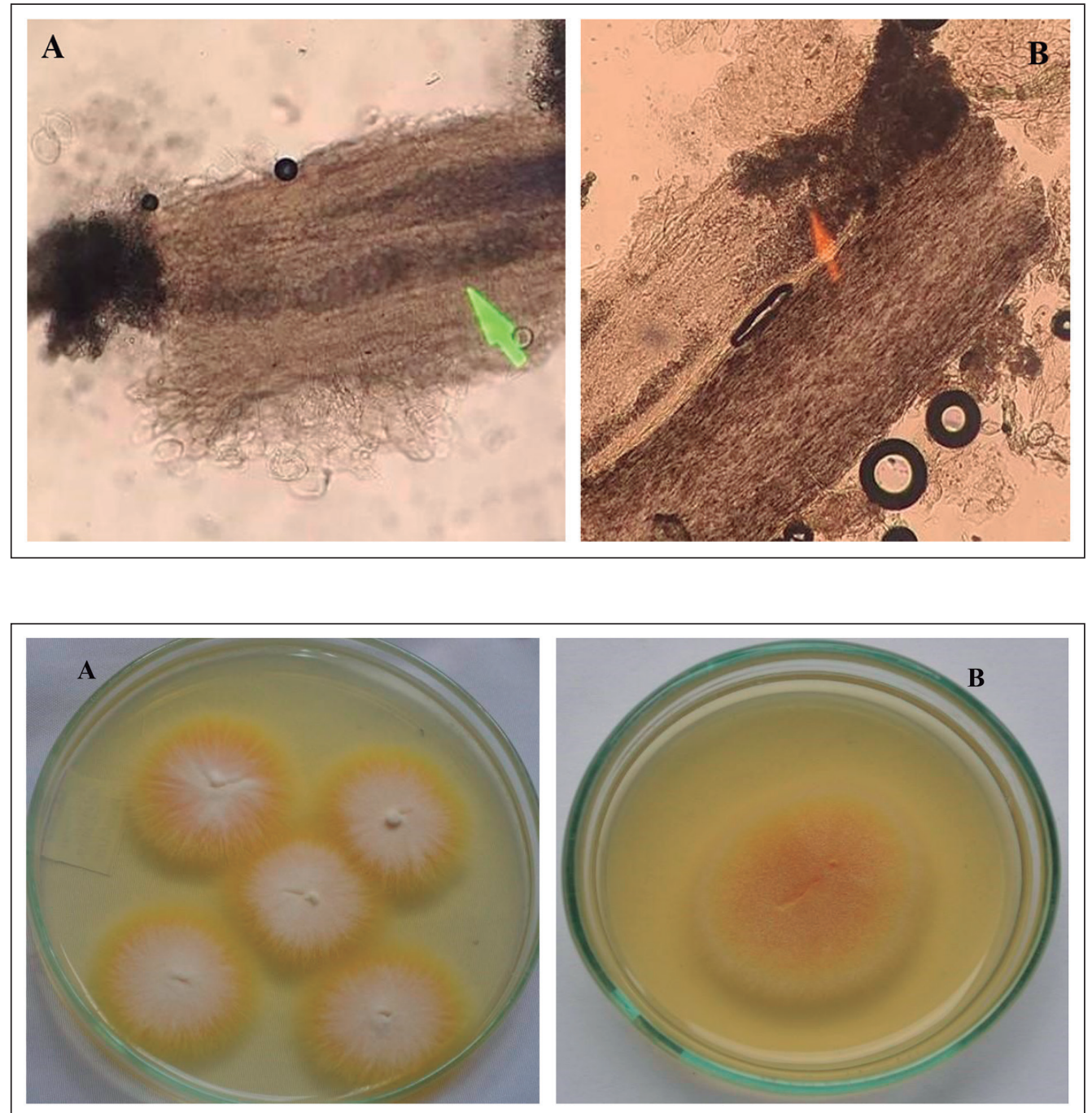

Figura 5. Hallazgos al examen microscópico directo $(\mathrm{KOH})$ en tiña capitis. A. Parasitación endotrix. B. Parasitación ectoendotrix del tallo piloso (Cortesía Dra. Janeth Villanueva. Laboratorio Micología Sección de Dermatología Universidad del Valle).

Figura 6. Cultivo micológico. A. Microsporum canis en agar Sabouraud. B. Trichophyton tonsurans en agar mycosel. Fotografías tomadas de la Colección de Microorganismos de la Escuela de Microbiología, Universidad de Antioquia (CM-EMUDEA). Cortesía: Diana Marcela González Gil, Microbióloga, MSc. Grupo de Investigación en Microbiología Básica y Aplicada - MICROBA. 
El estudio histopatológico usualmente demuestra la presencia de artrosporas en los folículos pilosos o en el estrato córneo, se puede visualizar crecimiento de hifas y formación de artroconidias en la superficie del pelo (ecto-endotrix) o dentro del tallo (endotrix). En la tiña fávica las hifas se encuentran en el estrato córneo, en el tallo y en el escudete o "cazoleta», hay atrofia folicular e infiltrado inflamatorio crónico en dermis. En el querion de Celso, puede observarse un infiltrado perifolicular superficial y/o profundo, rico en neutrófilos, eosinófilos y linfocitos asociado con secreción purulenta, destrucción folicular e inflamación granulomatosa ${ }^{7}$.

\section{Diagnóstico diferencial}

El diagnóstico diferencial varía según la presentación clínica, los hallazgos al examen físico y la evaluación con las herramientas diagnósticas previamente descritas. Se debe diferenciar la TC de otras entidades clínicas que pueden causar alopecia, hipotricosis y/o descamación en el cuero cabelludo en la edad pediátrica. Dentro de las patologías que deben diferenciarse de la TC se encuentran principalmente la alopecia areata y la tricotilomanía.

La tricotilomanía (TTM) constituye un trastorno del control de los impulsos caracterizado por el hábito recurrente de tracción y auto arrancamiento del pelo (del cuero cabelludo, cejas y/o de otras áreas corporales), generando zonas de hipotricosis o placas alopécicas irregulares no cicatriciales constituidas por pelos cortos de longitud variable, afectando principalmente la región parieto-occipital y el vertex ${ }^{40,41}$. Los rasgos tricoscópicos característicos de la TTM incluyen pelos fragmentados irregulares, enrollados, tricoptilosis (puntas abiertas), signo en $\mathrm{V}$ (dos pelos fragmentados de igual longitud que surgen de un mismo folículo), pelos "en espiral", "en llama" (restos de tallos pilosos proximales), "en tulipán” (terminaciones oscuras con forma de tulipán) y puntos negros irregulares ${ }^{42,43}$.

Por otra parte la alopecia areata, una patología de etiología autoinmune, se manifiesta con parches alopécicos ovalados y/o difusos, que a diferencia de la TC carece de cambios descamativos, y presenta hallazgos dermatoscópicos característicos tales como pelos en signo de exclamación (pelos cortos con extremos proximales afilados), vellos cortos y puntos amarillos ${ }^{32,44,45}$.

Otras patologías que deben diferenciarse de la TC incluyen la psoriasis del cuero cabelludo, una patología inflamatoria crónica que puede presentarse con placas eritematosas, descamativas, bien delimitadas generalmente no alopécicas con compromiso frecuente en otras áreas del cuerpo, como codos, rodillas y altera- ciones ungueales, permitiendo así su diferenciación de infecciones por dermatofitos ${ }^{46}$.

La dermatitis seborreica se caracteriza también por placas eritematosas, descamativas, pruriginosas localizadas en áreas seborreicas, como el cuero cabelludo, sin embargo, su compromiso difuso y su baja asociación con placas alopécicas permiten su diferenciación ${ }^{32,44}$. La falsa tiña o pitiriasis amiantácea es una condición eccematosa del cuero cabelludo, caracterizado por escamas gruesas, color asbesto, adheridas al pelo; siendo un diagnostico diferencial de la TC forma favosa ${ }^{47}$.

Finalmente, la forunculosis y el impétigo del cuero cabelludo son infecciones bacterianas que pueden confundirse con formas inflamatorias de la TC, pero se diferencian por la ausencia de antecedentes epidemiológicos, el curso más agudo y la respuesta al tratamiento antibiótico ${ }^{32}$.

\section{Tratamiento}

Los objetivos del tratamiento de TC incluyen la erradicación del organismo (curación clínica y micológica), el alivio de los síntomas, la prevención de alopecia cicatrizal y la reducción de la transmisión a otras personas. Para lograr estos objetivos, en la mayoría de los casos, se requiere de tratamiento sistémico ${ }^{4,16}$. Este tratamiento incluye consideraciones de eficacia, tolerabilidad, disponibilidad y costo de los medicamentos ${ }^{48}$ debiendo tener en consideración además la sospecha y/o confirmación micológica del agente causal, como se expone en la tabla 4.

Tradicionalmente, la griseofulvina se ha considerado de elección en la población infantil debido a su buen perfil de seguridad y pocos efectos secundarios $^{3,19}$. Como alternativas terapéuticas, itraconazol, griseofulvina y terbinafina, han mostrado las tasas más altas de curación ${ }^{5}$.

En las Infecciones por Microsporum spp, griseofulvina ha demostrado una mayor eficacia que terbinafina. Los resultados de una revisión sistemática y un metaanálisis de ensayos clínicos aleatorizados, describieron una tasa de curación más baja para TC por Microsporum tratadas con terbinafina durante seis semanas, en comparación con la terapia con griseofulvina, por un periodo de 6 a 12 semanas ( 34.7 versus 50,9 por ciento, RR 0,68, 95\% IC 0,53-0,86) ${ }^{49}$.

En las Infecciones por Trichophyton spp, tanto la griseofulvina como la terbinafina son considerados antimicóticos de primera línea ${ }^{49}$. Algunos estudios han demostrado una mayor eficacia de terbinafina, en comparación con griseofulvina, cuando se administran durante un período similar. Los datos de dos ensayos clínicos aleatorizados, que compararon un ciclo de seis semanas de gránulos de terbinafina $(5 \mathrm{a} 8 \mathrm{mg} / \mathrm{kg}$ por 
Tiña Capitis - N. Vargas-Navia et al

Tabla 4. Tratamiento según el tipo de parasitación de la tiña capitis

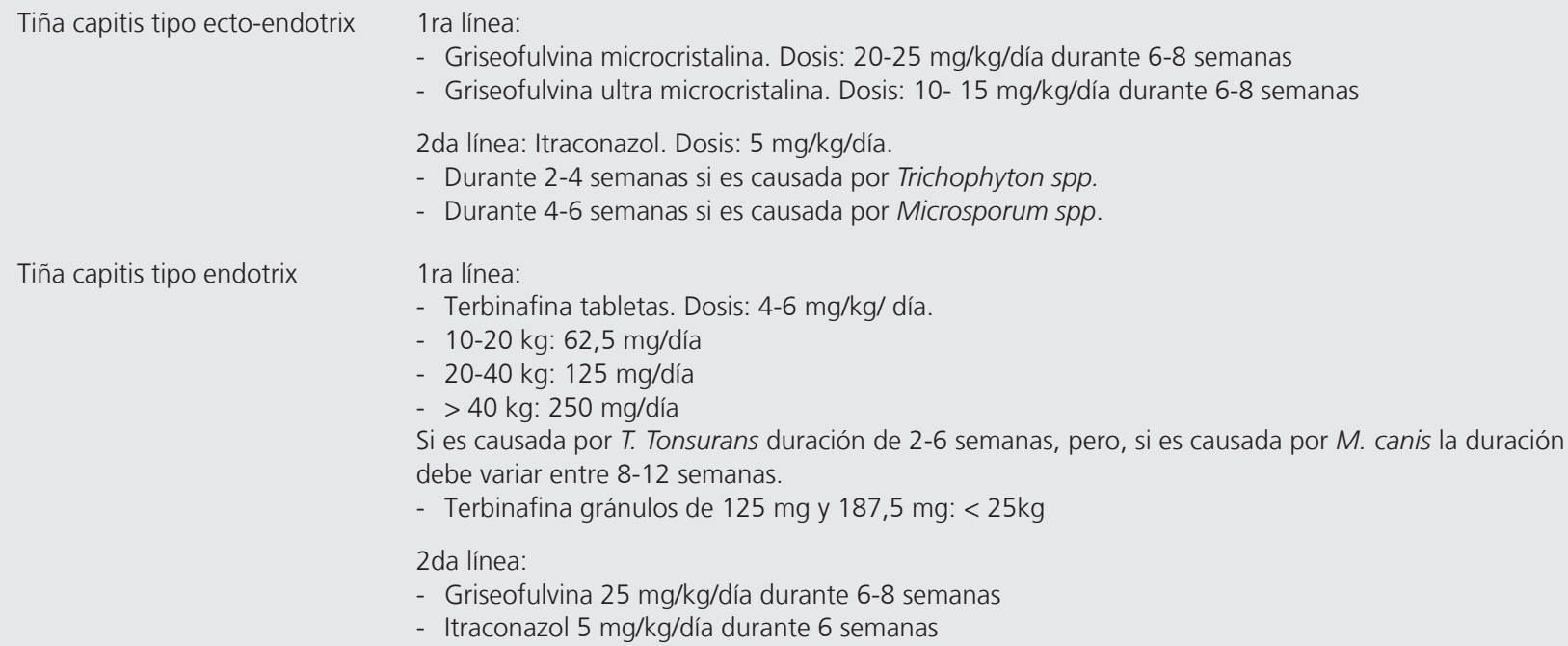

Adaptado y modificado de: Gupta AK, et al. $2018^{6}$.

día) con un ciclo de seis semanas de griseofulvina (10 a $20 \mathrm{mg} / \mathrm{kg}$ por día), encontraron una eficacia similar para la TC en general, pero mayor eficacia de la terbinafina para el subgrupo de niños con infecciones por T.tonsurans (tasa de curación completa 52,1 versus 35,4 por ciento, RR 1,47, IC 95\% 1,22-1,77). No hubo diferencia en el efecto de las infecciones por T. violaceum ${ }^{50}$.

La administración de griseofulvina está aprobada por la FDA (Food and Drug Administration) en niños mayores de 2 años, no se requieren pruebas de laboratorio de las enzimas hepáticas séricas si la duración del tratamiento no excede las 8 semanas. La terbinafina (gránulos) está aprobado por la FDA en niños mayores de 4 años, para tratamientos de 6 semanas, pero se recomienda una evaluación inicial y periódica de las enzimas hepáticas en sangre, cada 4 a 6 semanas $^{51}$. La dosificación de gránulos es mayor que la recomendada tradicionalmente para las tabletas, lo que refleja el hallazgo de que el aclaramiento de la droga con terbinafina es mayor en los niños ${ }^{48}$.

El tratamiento tópico no se recomienda como monoterapia ${ }^{4}$, sin embargo, el uso de antifúngicos tópicos como el ketoconazol al $2 \%$, sulfuro de selenio y ciclopirox olamina en champú, por 5 a 10 minutos, 2 a 3 veces por semana durante 15 días, incluso hasta la cura clínica y micológica, resulta útil como medida coadyuvante de la terapia oral y permite disminuir la tasa de transmisión de las especies causantes de la infección.

En relación con el manejo de fómites, es importante tener precaución al realizar cortes de pelo, afeitar la cabeza o compartir peinetas y gorros. Se recomienda que los objetos contaminados sean desinfectados con hipoclorito sódico o agua caliente con jabón ${ }^{3,5}$. En casos de infecciones causadas por especies zoofílicas, debe solicitarse la respectiva evaluación veterinaria de las mascotas en contacto con los menores infectados. La inasistencia a actividades escolares de los pacientes pediátricos con TC no es una recomendación práctica, ya que la diseminación de las esporas puede continuar durante meses a pesar de estar recibiendo tratamiento oral ${ }^{5}$.

En conclusión, la TC es una patología infecciosa que afecta frecuentemente a la población pediátrica. Un diagnóstico oportuno evita secuelas permanentes que afecten la calidad de vida. El aislamiento del agente causal permite orientar el tratamiento antifúngico adecuado, siendo estos eficaces y seguros en población pediátrica.

\section{Conflicto de intereses}

Los autores declaran no tener conflicto de intereses.

\section{Agradecimientos}

Dra. Carolina Palacio, Dermatóloga Especialista en Tricología. Universidad Militar Nueva Granada. Bogotá Colombia. Diana Marcela González Gil, Microbióloga, MSc. Grupo de Investigación en Microbiología Básica y Aplicada - MICROBA. Universidad de Antioquia, Medellín, Colombia. Dra. Janeth Villanueva, Laboratorio Micología Sección de Dermatología Universidad del Valle. 


\section{Referencias}

1. Gómez Moyano E, Crespo Erchiga V, Martínez Pilar L. Dermatofitosis. Piel. 2016;31(8):546-59.

2. Kechia FA, Kouoto EA, Nkoa T, et al. Epidemiology of tinea capitis among school-age children in Meiganga, Cameroon. J Mycol Med [Internet]. 2014;24(2):129-34. Available from: http:// dx.doi.org/10.1016/j.mycmed.2013.12.002

3. Zuluaga A, Cáceres DH, Arango K, de Bedout C, Cano LE. Epidemiología de la tinea capitis: 19 años de experiencia en un laboratorio clínico especializado en Colombia. Infectio [Internet]. 2016;20(4):225-30. Available from: http:// dx.doi.org/10.1016/j.infect.2015.11.004

4. Fuller LC, Barton RC, Mohd Mustapa MF, Proudfoot LE, Punjabi SP, Higgins EM. British Association of Dermatologists' guidelines for the management of tinea capitis 2014 . Br J Dermatol. 2014;171(3):454-63.

5. A BTJB. Micología médica básica. 5ta edició. Bonifaz A, editor. México DF: México: McGraw Hill; 2015. 108-58 p.

6. Gupta AK, Mays RR, Versteeg SG, et al. Tinea capitis in children: a systematic review of management. Vol. 32, Journal of the European Academy of Dermatology and Venereology. 2018. 2264-2274 p.

7. Rebollo N, López-Barcenas AP, Arenas R. Tiña de la cabeza. Actas Dermosifiliogr. 2008;99(2):91-100.

8. Aranibar Duran L, Stevens González J, Palma Ducommun C, Zapata Manque S, Pizarro Olave J. Estudio epidemiológico de la tinea capitis en una población de Santiago de Chile. La aparición de Trichophyton tonsurans como agente etiológico. Piel. 2017;32(10):604-9.

9. Abdel-Rahman SM, Farrand N, Schuenemann E, et al. The prevalence of infections with Trichophyton tonsurans in schoolchildren: The CAPITIS study. Pediatrics. 2010;125(5):966-73.

10. Balci E, Gulgun M, Babacan O, et al. Prevalence and risk factors of tinea capitis and tinea pedis in school children in Turkey. J Pak Med Assoc. 2014;64(5):5148.

11. Santos PE, Córdoba S, Rodero LL, Carrillo-Muñoz AJ, Lopardo HA. Tinea capitis. Experiencia de 2 años en un hospital de pediatría de Buenos Aires, Argentina. Rev Iberoam Micol. 2010;27(2):104-6.

12. John AM, Schwartz RA, Janniger CK. The kerion: An angry tinea capitis. Int J Dermatol. 2018;57(1):3-9.

13. Bassyouni RH, El-Sherbiny NA, Abd El Raheem TA, Mohammed BH. Changing in the epidemiology of tinea capitis among school children in Egypt. Ann Dermatol. 2017;29(1):13-9.
14. Gits-Muselli M, Benderdouche M, Hamane $\mathrm{S}$, et al. Continuous increase of Trichophyton tonsurans as a cause of tinea capitis in the urban area of Paris, France: A 5-year-long study. Med Mycol. 2017;55(5):476-84.

15. Fuller LC. Changing face of tinea capitis in Europe. Curr Opin Infect Dis. 2009;22(2):115-8.

16. Bhat $\mathrm{Y}$, Zeerak S, Kanth F, Yaseen A, Hassan I, Hakak R. Clinicoepidemiological and mycological study of tinea capitis in the pediatric population of kashmir valley: A study from a tertiary care centre. Indian Dermatol Online J. 2017;8(2):100.

17. Zampella JG, Kwatra SG, Blanck J, Cohen B. Tinea in Tots: Cases and Literature Review of Oral Antifungal Treatment of Tinea Capitis in Children under 2 Years of Age. J Pediatr [Internet]. 2017;183:1218.e3. Available from: http://dx.doi. org/10.1016/j.jpeds.2016.12.042

18. Magill SS, Manfredi L, Swiderski A, Cohen B, Merz WG. Isolation of Trichophyton violaceum and Trichophyton soudanense in Baltimore, Maryland. J Clin Microbiol. 2007;45(2):461-5.

19. Marcoux D, Dang J, Auguste H, et al. Emergence of African species of dermatophytes in tinea capitis: A 17-year experience in a Montreal pediatric hospital. Pediatr Dermatol. 2018;35(3):323-8.

20. Frías-DeLeón MG, Porras-López C, Martínez-Villarreal JD, et al. Tinea capitis. Datos epidemiológicos actuales en una región subtropical de Mesoamérica (Guatemala). Dermatologia Rev Mex. 2017;61(5):371-8.

21. Mayorga J, Esquivel-González PL, PradoTrillo A, Barba-Gómez JF. Características clínicas y epidemiológicas de pacientes con infección por Microsporum canis. Dermatologia Rev Mex. 2016;60(1):18-23.

22. Veasey JV, Miguel BAF, Mayor SAS, Zaitz C, Muramatu LH, Serrano JA. Epidemiological profile of tinea capitis in São Paulo City. An Bras Dermatol. 2017;92(2):283-4.

23. Arenas R, Torres E, Amaya M, et al. Emergence of Microsporum audouinii and Trichophyton tonsurans as Causative Organisms of Tinea Capitis in the Dominican Republic. Actas Dermo-Sifiliográficas (English Ed. 2010;101(4):330-5.

24. Benavides J, Villanueva J. Tiña del cuero cabelludo por Trichophyton tonsurans: agente emergente en Colombia. Rev Asoc Colomb Dermatol. 2010;18:99-101.

25. Moreno Morales M, Valdez Landrum P, García Valdés A, Arenas Guzmán R. Brote epidémico intrafamiliar de tiña de la cabeza por Trichophyton tonsurans: informe de cuatro casos en tres generaciones. Med Cutan Ibero Lat Am. 2015;43(3):217-21.

26. Garc L, Espinosa-ruiz JJ. Tiña capitis por Microsporum gypseum, una especie infrecuente. Arch Argent Pediatr. 2018;116(2):296-9.

27. López-Estebaranz JL, Sopena-Barona J. Cutaneous dermatophytoses. Etiology, epidemiology and clinical manifestations. Med Clin (Barc). 2006;126(SUPPL.1):14-9.

28. Niyonsaba F, Ogawa H. Protective roles of the skin against infection: Implication of naturally occurring human antimicrobial agents -defensins, cathelicidin LL-37 and lysozyme. J Dermatol Sci. 2005;40(3):15768.

29. Mao L, Zhang L, Li H, et al. Pathogenic fungus microsporum canis activates the NLRP3 inflammasome. Infect Immun. 2014;82(2):882-92.

30. Calderon RA, Hay RJ. Fungicidal activity of human neutrophils and monocytes on dermatophyte fungi, Trichophyton quinckeanum and Trichophyton rubrum. Immunology. 1987;61(3):289-95.

31. Arenas R. Dermatofitosis . Capítulo 6. Micología médica ilustrada. 5.a Edició. México DF; 2014. 67-98 p.

32. Hay RJ. Tinea Capitis: Current Status. Mycopathologia. 2017;182(1-2):87-93.

33. Torres-Guerrero E, Martínez-Herrera E, Arroyo-Camarena S, Porras C, Arenas R. Kerion Celsi: A report of two cases due to Microsporum gypseum and Trichophyton tonsurans. Our Dermatology Online. 2015;6(4):424-7.

34. Ekiz Ö, en BB, Rifaio lu EN, Balta I. Trichoscopy in paediatric patients with tinea capitis: A useful method to differentiate from alopecia areata. J Eur Acad Dermatology Venereol. 2014;28(9):1255-8.

35. Elghblawi E. Tinea Capitis in Children and Trichoscopic Criteria. Int J Trichol. 2017;9:47-9.

36. Arrazola-Guerrero J, Isa-Isa R, TorresGuerrero E, Arenas R. Tiña de la cabeza: Descripción de los hallazgos dermatoscópicos en 37 pacientes. Rev Iberoam Micol. 2015;32(4):242-6.

37. Brasileiro A, Campos S, Cabete J, Galhardas C, Lencastre A S V. Trichoscopy as an additional tool for the differential diagnosis of tinea capitis: a prospective clinical study. Br J Dermatol. 2016;175(1):208-9.

38. Aqil N, Baybay H, Moustaide K, Douhi Z, Elloudi S, Mernissi FZ. A prospective study of tinea capitis in children: Making the diagnosis easier with a dermoscope. J Med Case Rep. 2018;12(1):1-7.

39. Wa kiel-Burnat A, Rakowska A, Sikora $\mathrm{M}$, Ciechanowicz P, Olszewska M, Rudnicka L. Trichoscopy of Tinea Capitis: 
A Systematic Review. Dermatol Ther (Heidelb). 2020;10(1):43-52.

40. Khitam Al-Refu. Clinical Significance of Trichoscopy in Common Causes of Hair Loss in Children: Analysis of 134 Cases. Int J Trichology. 2018;10(4):154-161.

41. Xu L, Liu KX, Senna MM. A Practical Approach to the Diagnosis and Management of Hair Loss in Children and Adolescents. Front Med 2017 24;4

42. Khunkhet S, Vachiramon V, Suchonwanit P. Trichoscopic clues for diagnosis of alopecia areata and trichotillomania in Asians. Int J Dermatol. 2017;56(2):161-5.

43. Martín JM, Montesinos E, Cordero P, Gonzalez V, Ramon D. Trichoscopy features of trichotillomania. Pediatr Dermatol. 2019;36(2):265-7.
44. Hawkins DM, Smidt AC. Superficial Fungal Infections in Children. Pediatr Clin North Am. 2014;61(2):443-55.

45. El-Taweel AE, El-Esawy F, Abdel-Salam O. Different trichoscopic features of tinea capitis and alopecia areata in pediatric patients. Dermatol Res Pract. 2014;2014.

46. Gupta AK, MacLeod MA, Foley KA, Gupta G, Friedlander SF. Fungal skin infections. Pediatr Rev. 2017;38(1):8-22.

47. Errichetti E, Stinco G. Dermoscopy as a useful supportive tool for the diagnosis of pityriasis amiantacea-like tinea capitis. Dermatol Pract Concept. 2016;6(3):63-5.

48. Kimberlin DW. Red Book (2018): Report of the Committee on Infectious Diseases. 31st ed. Elk Grove Village: American
Academy of Pediatrics; 2018. 1153 p.

49. Chen X, Jiang X, Yang M, et al. Systemic antifungal therapy for tinea capitis in children: An abridged Cochrane Review. J Am Acad Dermatol. 2017;76(2):368-74.

50. Elewski BE, Cáceres HW, DeLeon $\mathrm{L}$, et al. Terbinafine hydrochloride oral granules versus oral griseofulvin suspension in children with tinea capitis: Results of two randomized, investigatorblinded, multicenter, international, controlled trials. J Am Acad Dermatol. 2008;59(1):41-54.

51. Patel D, Castelo-Soccio LA, Rubin AI, Streicher JL. Laboratory monitoring during systemic terbinafine therapy for pediatric onychomycosis. JAMA Dermatology. 2017;153(12):1326-7. 